\title{
The effect of fumigation with microencapsulated allyl isothiocyanate in a gas-barrier bag against Solenopsis invicta (Hymenoptera: Formicidae)
}

\author{
Yoshiaki Hashimoto ${ }^{1} \cdot$ Hironori Sakamoto ${ }^{2} \cdot$ Hiromi Asai $^{3} \cdot$ Masamitsu Yasoshima ${ }^{4} \cdot$ Hui-Min Lin $^{5} \cdot$ Koichi Goka $^{2}$
}

Received: 17 February 2020 / Accepted: 8 April 2020 / Published online: 23 April 2020

(c) The Author(s) 2020

\begin{abstract}
Red imported fire ants, Solenopsis invicta Buren (Hymenoptera: Formicidae), which are among the most harmful alien ants, were first detected in Japan in a shipping container from China in May 2017, and continue to enter Japan via this trade pathway. Fumigation is one of the most effective ways to eliminate $S$. invicta from goods transported in containers. The present study is the first to evaluate the effect of fumigation with allyl isothiocyanate (AITC) against S. invicta. Fumigation using microencapsulated AITC pellets in a gas-barrier bag for $24 \mathrm{~h}$ completely killed $S$. invicta. Furthermore, our fumigation system was able to maintain a high concentration of AITC vapor for at least 13 days. AITC, a naturally occurring compound in wasabi [Eutrema japonicum (Miq.) Koidz], has been demonstrated to be safe for humans and the environment, but its use as a fumigant is limited by its strong volatility and pungency. However, its encapsulation solves this problem by allowing controlled vapor release. Thus, the use of microencapsulated AITC in combination with a gas-barrier bag has considerable potential to provide an effective and easy-to-use protocol for fumigation of S. invicta.
\end{abstract}

Keywords Red imported fire ant $\cdot$ Wasabi $\cdot$ Allyl isothiocyanate $\cdot$ Fumigant $\cdot$ Invasive alien species

\section{Introduction}

Red imported fire ant, Solenopsis invicta Buren (Hymenoptera: Formicidae), is one of the most harmful invasive species and poses serious hazards to agriculture, natural

Electronic supplementary material The online version of this article (https://doi.org/10.1007/s13355-020-00684-9) contains supplementary material, which is available to authorized users.

Yoshiaki Hashimoto

yoshiaki@hitohaku.jp

1 Institute of Natural and Environmental Sciences, University of Hyogo/The Museum of Nature and Human Activities, Hyogo, Yayoigaoka 6, Sanda, Hyogo 669-1546, Japan

2 Center for Environmental Biology and Ecosystem Studies, National Institute for Environmental Studies, Onogawa 16-2, Tsukuba, Ibaraki 305-8506, Japan

3 PRD Co., Ltd., Takaramachi 12-3, Higashiosaka, Osaka 579-8025, Japan

4 Moriya Industry, Hanatennishi 3-10-7, Joto, Osaka 536-0011, Japan

5 Monsters' Agrotech, 1F., No.285, Sec. 2, Tingzhou Rd., Zhongzheng Dist., Taipei City 100, Taiwan environments, and public health (Wylie and Janssen-May 2016). In Japan, S. invicta was first discovered in a shipping container from China in Amagasaki, Hyogo Prefecture, in May 2017, and the invasion into Japan via this trade pathway is continuing (Ministry of Environment Japan 2020). By the end of January 2020, 48 independent incursions of S. invicta had been detected in 15 prefectures of Japan, 28 of which were found in containerized cargo unloaded in ports or delivered to customers. Fortunately, these invasions were successfully intercepted and the ants were eradicated. However, the risk of S. invicta establishing in Japan rises with the increase in the invasion pressure associated with containerized cargo traffic (Wylie et al. 2020).

Fumigation is one of the quickest and most effective ways to eliminate pests from goods transported in containers (Rajendran 2004). Synthetic pesticides, such as methyl bromide and phosphine, are the most widely used chemicals to fumigate containerized cargo (Zettler and Arthur 2000). However, $S$. invicta is introduced into Japan with various transported goods, including stationery goods, tableware, blankets, and animal feedstuff (Ministry of Environment Japan 2020). Synthetic fumigants may be inappropriate for such non-mechanical products because of residual toxicity. 
Therefore, there is an urgent need to develop safe alternative fumigants for $S$. invicta to replace synthetic pesticides. Moreover, in Japan, imported goods are rapidly moved from ports of entry and delivered to customers within a few days. For example, in the first incursion of $S$. invicta in Japan, the cargo contaminated with the ants was delivered from Kobe port to a warehouse in an inland city, Amagasaki, within 4 days (Ministry of Environment Japan 2020). Safe use of synthetic fumigants requires special facilities and equipment, such as a vacuum pump and chamber, gas introduction and exhaust system, and protective clothing and gear, which are expensive and not readily available (Rajendran 2004). Therefore, to quickly eliminate $S$. invicta delivered to customers and to prevent its spread into inland Japan, the development of a safer and simpler fumigation method is urgently needed.

Allyl isothiocyanate (AITC) is a naturally occurring compound from plants, such as wasabi [Eutrema japonicum (Miq.) Koidz]. The vapor of AITC is well recognized as a strong fumigant for the control of insect pests, and its safety for humans and environment has been demonstrated (Tsao et al. 2002; Worfel et al. 1997; Wu et al. 2009). Especially, unlike synthetic fumigants, AITC is a gas that has a low water solubility and easy degradation in the atmosphere (Weerawatanakorn et al. 2015). Such properties make AITC an environmental and human safety fumigant without pesticide residue concerns (US EPA 2013). However, its use as a fumigant is limited by its strong volatility and pungency. In addition, AITC is usually sold in liquid form and requires the addition of a setting treatment to use it as a fumigant. Recently, microencapsulation technology and applications of AITC using spray-drying and polyethylene have been established (Patent No. JP5033232B). This technology enables the sustained release of AITC through semi-permeable capsule membranes, which can decrease irritancy by preventing the excessive release. Additionally, the microencapsulation can convert liquid AITC into pelletor sheet-like polyethylene-based material, which allows easy handling of AITC when it is used as a fumigant. Therefore, although the microencapsulated AITC has not been used as a fumigant against $S$. invicta, it has a good potential as a safe and effective solution to sanitize cargo and eradicate the invading pest. Our previous study, which used microencapsulated AITC and bait traps, found that AITC not only completely prevented $S$. invicta from accessing the bait but also killed the workers that failed to escape from the traps (Hashimoto et al. 2019). Therefore, in the present study, we used microencapsulated AITC and a gas-barrier bag in fumigation experiments to evaluate the insecticidal activity of AITC against $S$. invicta. Furthermore, a fumigation only works properly if an adequate concentration can be maintained for an adequate exposure period. Therefore, we measured the changes in AITC concentration in the bag to estimate the efficacy of our fumigation system. This study may provide a new and useful fumigation method to prevent S. invicta invasion to Japan.

\section{Materials and methods}

\section{Insect materials}

Solenopsis invicta workers were collected from a nest in Tucheng District, New Taipei City, Taiwan $\left(23624^{\circ} 58^{\prime} 41.1^{\prime \prime} \mathrm{N}, 121^{\circ} 26^{\prime} 49.6^{\prime \prime} \mathrm{E}\right)$. The ants were kept in a plastic tray containing a piece of damp cotton wool and reared on honey water (ca. 10\%) in a room at around $25{ }^{\circ} \mathrm{C}$ and $70 \%$ RH in Taipei City, Taiwan.

\section{Fumigation materials}

As a fumigant, we used $50 \mathrm{~g}$ of microencapsulated AITC pellets containing in total $1 \mathrm{~g}$ of AITC (the weight of each pellet was $0.01 \mathrm{~g}$, and contained $2 \%$ of AITC by weight); the pellets were obtained from PRD Co., Ltd. (Osaka, Japan). A transparent gas-barrier bag $(91 \mathrm{~cm}$ width $\times 60 \mathrm{~cm}$ length $\times 40 \mathrm{~cm}$ depth, $80 \mathrm{~L}$ ) used for fumigation consisted of a poly(ethylene terephthalate) film (12 $\mu$ m thickness) deposited on a film of linear low-density polyethylene $(80 \mu \mathrm{m}$ thickness). The gas-barrier bags were developed by Moriya Industry Co., Ltd. (Osaka, Japan).

\section{Fumigation treatments}

.For the ease of handling, 10 workers of S. invicta were placed in a polypropylene snap-cap vial $(50 \mathrm{~mL}, 3.5 \mathrm{~cm}$ diameter, and $7.3 \mathrm{~cm}$ depth) with a piece of damp cotton wool. The vial had a cap with a mesh-covered $3 \mathrm{~cm}$ diameter opening, which prevented the ants from escaping and allowed air to flow into the inside (Fig. 1a). For fumigation treatment, we placed the vial in a cardboard box $(15 \mathrm{~cm}$ width $\times 29 \mathrm{~cm}$ length $\times 13 \mathrm{~cm}$ depth) and then put the box in a gas-barrier bag with microencapsulated AITC pellets holding in a tea filter bag (Fig. 1b, c). In the control treatment, the bag contained no pellets. In both treatments, $S$. invicta were removed from the bag after $24 \mathrm{~h}$ of exposure, and mortality was determined by observation. Ants were considered dead if they did not respond to probing. They were considered moribund when locomotion was not possible and the movement of appendages was feeble and uncoordinated. We counted the number of moribund ants as alive but confirmed whether they showed any subsequent recovery 
Fig. 1 Test device used to evaluate the effect of fumigation with microencapsulated AITC against $S$. invicta. a Polypropylene snap-cap vials with a meshcovered $3-\mathrm{cm}$ diameter opening. Ten workers of $S$. invicta were placed in each of the vial with a piece of damp cotton wool. b Cardboard box used for the fumigation test. A polypropylene snap-cap vial containing $S$. invicta workers was put in the box. c Gas-barrier bag used in the fumigation test. A microencapsulated AITC pellet (50 g) and a cardboard box with a vial containing $S$. invicta were placed in the bag. $G B B$ gasbarrier bag, MAP microencapsulated AITC pellet. d Covered glass Petri dish used to observe the behavior of an S. invicta worker exposed to AITC. MAS microencapsulated AITC sheet, SI S. invicta worker
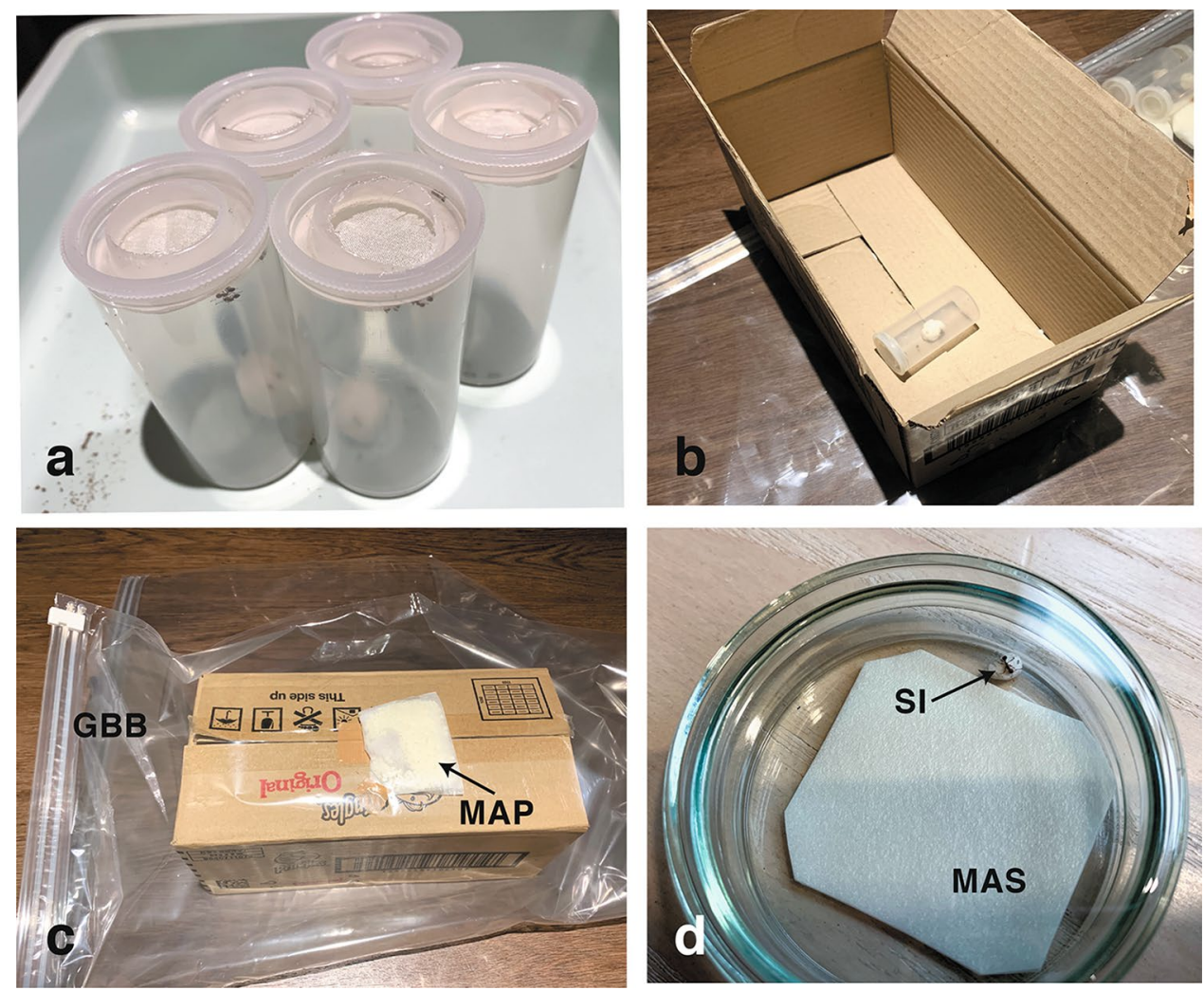

of activity during the next day. Using four gas-barrier bags, we performed two trials in each of the control and fumigation treatments daily and were repeated for 5 consecutive days (i.e., 10 trails per treatments).

\section{Statistical analysis}

For statistical analysis, to compare mortality rates between control and fumigation treatments, we used generalized linear mixed models (GLMM) with binomial distribution and a logistic regression link. To control for potential differences in gas-barrier properties among the bags and conditions of trial days, we included "bag differences" and "trail days" as random intercept effects in the GLMM. Analysis of the GLMM was performed using R package lme4 version 1.1-21 (Bates et al. 2015; R Core Team 2019).

\section{AITC release kinetics in the gas-barrier bag}

To evaluate the ability of the gas-barrier bag to maintain AITC vapor, we measured the kinetics of AITC release from the pellets in the bag. As in the fumigation treatment, we put $50 \mathrm{~g}$ of the microencapsulated AITC pellets together with a cardboard box containing a piece of damp cotton wool into a gas-barrier bag, but without $S$. invicta. Release kinetics were measured using the colorimetric gas detector tube (No.149, Gastec Corp., Ayase, Japan; measuring range 5-500 ppm). The detector tubes are graduated glass tubes filled with chemical reagents that produce a color stain when exposed to the target gas. The length of the color change is proportional to the concentration of the gas being tested (Noda et al. 2001). Gas in the bag was pulled out by a gas sampling pump (Ap-1, Komyo Rikagaku Kogyo KK, Japan) and passed through a detector tube with a needle from a hole in the bag. The hole was tightly closed with sealing tape to avoid gas leakage during the experiment. Gas samples were taken at 1, 3, 6, and $24 \mathrm{~h}$ and 2, 5, and 13 days. The measurements had three replicates. We assessed AITC release kinetics in a laboratory of PRD Co., Ltd. in Osaka City, Japan, at around $25^{\circ} \mathrm{C}$ and $70 \% \mathrm{RH}$.

\section{Behavior observation}

We observed ant behavior in responses to the vapor. A single worker of S. invicta and a microencapsulated AITC sheet (40 $\mathrm{mm} \times 40 \mathrm{~mm}, 1 \mathrm{~mm}$ thick, AITC content $0.01 \mathrm{~g}$ ) was placed in a covered glass Petri dish (64 $\mathrm{mm}$ diameter, $20 \mathrm{~mm}$ height) with a piece of damp cotton wool (Fig. 1d). The behavior of the worker within the arena was observed and 
video-recorded on a smartphone camera (iPhone XS produced by Apple Inc, USA).

\section{Results and discussion}

Microencapsulated AITC pellets showed strong and rapid (after only $24 \mathrm{~h}$ exposure) insecticidal activity against $S$. invicta when used as a fumigant in a gas-barrier bag (Fig. 2). The mean mortality rate in the fumigation treatment was 0.89 (95\% CI 0.81-0.94), which was significantly higher than the control treatment (mean 0.13 , 95\% CI 0.08-0.21) (GLMM, $\chi^{2}=83.61, d f=1, p<0.001$, Table S1). All survivors in the fumigation treatment were in the moribund state and died within the next day, which indicated that the treatment completely killed $S$. invicta. We also directly observed that a worker of $S$. invicta was quickly paralyzed and knocked down for only 2-h exposure to AITC (see Supplementary Movies 1, 2, 3, and 4). These results indicated that the microencapsulated AITC is a good candidate for an effective fumigant against $S$. invicta. However, we tested the efficiency of AITC only against $S$. invicta workers, not against the queens. A fumigation test indicated that both of the queens and workers of $S$. invicta were effectively controlled by ethyl formate, but queens were more tolerant to the fumigant than the workers (Lee et al. 2019). Therefore, to verify the efficacy of our fumigation method against $S$. invicta, the insecticidal effect of AITC against the queens needs to be further explored.

The evaluation of volatiles released from the microencapsulated AITC pellets $(50 \mathrm{~g})$ in a gas-barrier bag showed a quick rise in the concentration within $6 \mathrm{~h}$, and the value reached the range of 234-248 ppm $(n=3)$ at $24 \mathrm{~h}$ (Fig. 3). After 5 days, the concentration in the bag reached $500 \mathrm{ppm}$, which was the maximum that can be measured by the detector tube used, and the value was maintained for at least 13 days. Microencapsulated AITC has a high ability for a sustained release of the vapor through semi-permeable capsule membranes. For example, $5 \mathrm{~g}$ of the microencapsulated AITC pellets (AITC content $0.1 \mathrm{~g}$ ) can maintain $10 \mathrm{ppm}$ of the vapor in a cardboard box $(10 \mathrm{~cm} \times 10 \mathrm{~cm} \times 8 \mathrm{~cm})$ for $1 \mathrm{~h}$, without any gas-barrier cover or bag (our unpublished data). Consequently, in combination with a gas-barrier, microencapsulated AITC can control and maintain a high concentration of the vapor for a long period, which is a prerequisite for efficient fumigation. Because our method allowed maintaining a high concentration of AITC vapor, it might be used as an effective and easy fumigation method against $S$. invicta.

Overall, the results show that the fumigation method with microencapsulated AITC and a gas-barrier bag could be a feasible solution for S. invicta disinfestation because AITC

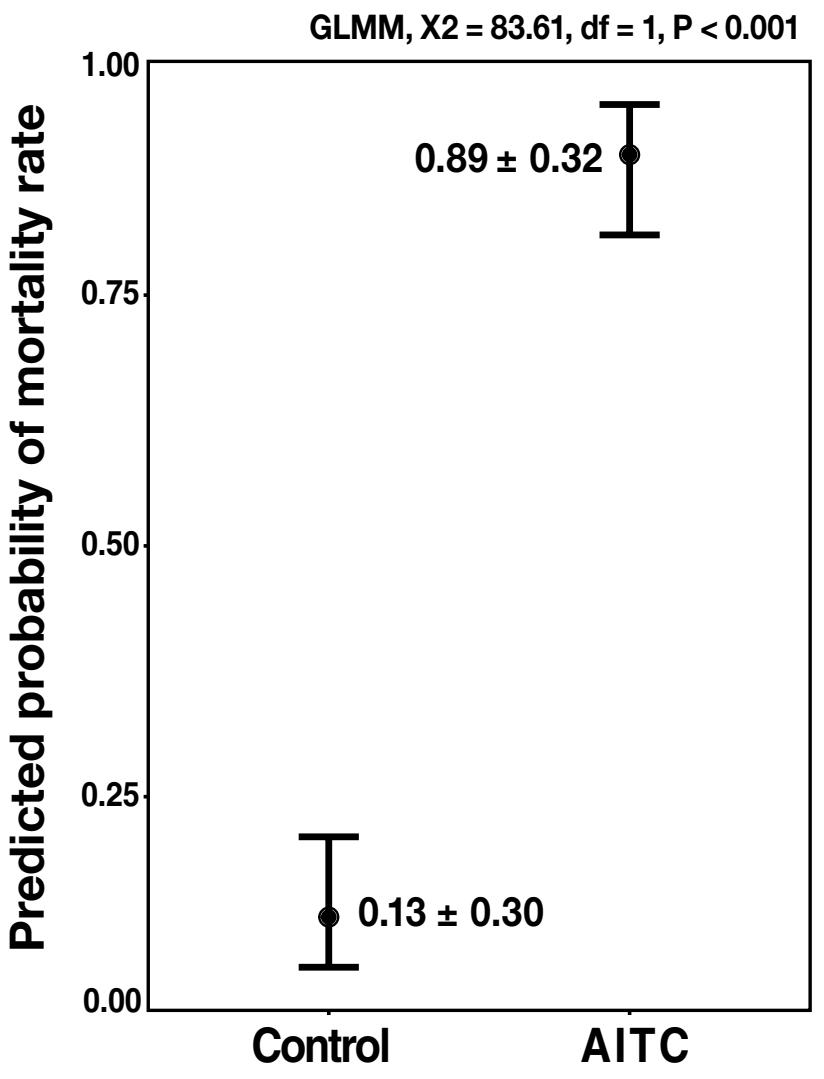

Fig. 2 Probabilities of mortality rates predicted by the GLMM for $S$. invicta workers exposed to AITC for $24 \mathrm{~h}$ and the control. Vertical lines show 95\% confidence intervals. Values shown in the graph are the mean \pm standard error of the mean

has a strong insecticidal effect on the ants and the system is easy to use. In addition, high volatility and degradability of AITC, coupled with moderate release property of its microencapsulation, contributes to the low residue content after fumigation (Weerawatanakorn et al. 2015; Worfel et al. 1997). However, for the practical application of our fumigation method, future research is needed to evaluate the efficacy in scaled-up spatial circumstances and against greater numbers of ants, including queens and workers. Furthermore, more detailed research, such as determination of the median lethal concentration $\left(\mathrm{LC}_{50}\right)$ for $S$. invicta, is required to determine the recommended dose of microencapsulated AITC in our fumigation system. However, AITC is a naturally occurring chemical considered harmless to human health and has been shown to inhibit the growth of fungi, insects, and bacteria (Cardiet et al. 2012; Meira et al. 2017; Torrijos et al. 2019). Thus, the development of our fumigation system will be significant not only for preventing of $S$. invicta invasion in Japan but also for pest management in museums and the food industry. 
Fig. 3 Concentration of AITC released from microencapsulated AITC pellets $(50 \mathrm{~g}$ in total) in a gas-barrier bag. Symbols indicated each measured value (replicates $=3$ ). The solid line connects the means

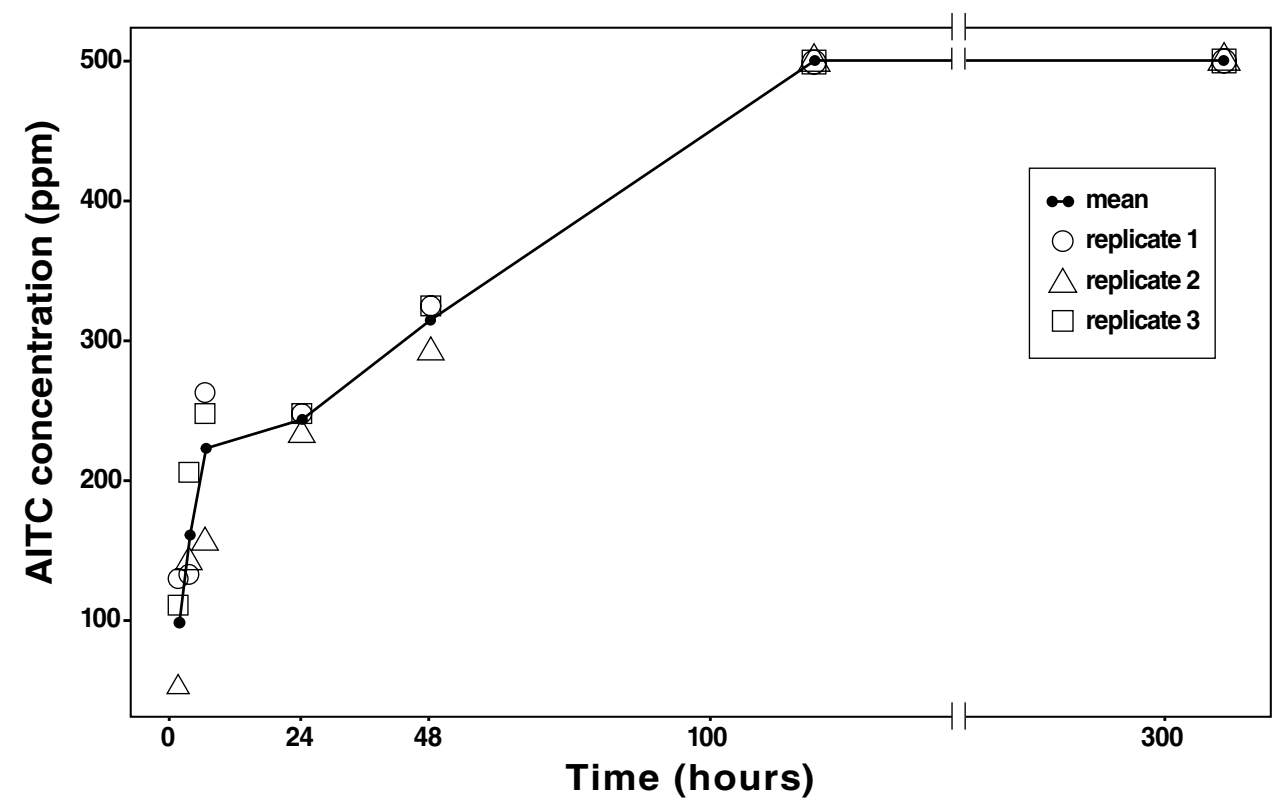

Acknowledgements We are thankful to the staff of Monsters' Agrotech for their valuable assistance in Taiwan. We also extend many thanks to Haruyuki Kinoshita (PRD Co., Ltd) for providing laboratory equipment. The present study could not have been accomplished without their kind assistance.

Funding This research received no specific grant from any funding agency.

\section{Compliance with ethical standards}

Conflict of interest The authors declare that they have no conflict of interest.

Open Access This article is licensed under a Creative Commons Attribution 4.0 International License, which permits use, sharing, adaptation, distribution and reproduction in any medium or format, as long as you give appropriate credit to the original author(s) and the source, provide a link to the Creative Commons licence, and indicate if changes were made. The images or other third party material in this article are included in the article's Creative Commons licence, unless indicated otherwise in a credit line to the material. If material is not included in the article's Creative Commons licence and your intended use is not permitted by statutory regulation or exceeds the permitted use, you will need to obtain permission directly from the copyright holder. To view a copy of this licence, visit http://creativecommons.org/licenses/by/4.0/.

\section{References}

Bates D, Mächler M, Bolker B, Walker S (2015) Fitting linear mixedeffects models using lme4. J Stat Softw 67:1-48

Cardiet G, Fuzeau B, Barreau C, Fleurat-Lessard F (2012) Contact and fumigant toxicity of some essential oil constituents against a grain insect pest Sitophilus oryzae and two fungi, Aspergillus westerdijkiae and Fusarium graminearum. J Pest Sci 85:351-358

Hashimoto Y, Yoshimura M, Huang RN (2019) Wasabi versus red imported fire ants: preliminary test of repellency of microencapsulated allyl isothiocyanate against Solenopsis invicta (Hymenoptera: Formicidae) using bait traps in Taiwan. Appl Entomol Zool 54:193-196

Lee BH, Park CG, Park MG, Roh GH, Kim D, Riddick EW, Chene J, Cha DH (2019) Ethyl formate fumigation for the disinfestation of red imported fire ants Solenopsis invicta Buren. J Asia Pac Entomol 22:838-840

Meira NV, Holley RA, Bordin K, de Macedo RE, Luciano FB (2017) Combination of essential oil compounds and phenolic acids against Escherichia coli O157: H7 in vitro and in dry-fermented sausage production. Int J Food Microbiol 260:59-64

Ministry of Environment Japan (2020). https://www.env.go.jp/natur e/intro/2outline/attention/hiari.html. Accessed 31 Jan 2020 (in Japanese)

Noda K, Naganawa R, Tao H, Sakurai K, Numata Y, Matsumoto S (2001) Simple analytical method for the determination of volatile chloroorganic compounds in water using lipid-coated quartz crystal microbalance sensor. IEEJ Trans Sens Micromach 121:388394 (in Japanese)

R Core Team (2019) R: a language and environment for statistical computing. R Foundation for Statistical Computing, Vienna, Austria. https://www.R-project.org/. Accessed 29 Feb 2020

Rajendran S (2004) Fumigation of shipping or freight containers. Outlooks Pest Manag 15:222-228

Torrijos R, Nazareth TM, Pérez J, Mañes J, Meca G (2019) Development of a bioactive sauce based on oriental mustard flour with antifungal properties for PITA bread shelf life improvement. Molecules 24:1019

Tsao R, Peterson CJ, Coats JR (2002) Glucosinolate breakdown products as insect fumigants and their effect on carbon dioxide emission of insects. BMC Ecol 2:5

US EPA US Environmental Protection Agency (2013) Oil of mustard and allyl isothiocyanate (AITC). http://www.regulation s.gov/\#!documentDetail;D=EPA-HQ-OPP-2013-0658-0006. Accessed $29 \mathrm{Feb} 2020$

Weerawatanakorn M, Wu JC, Pan MH, Ho CT (2015) Reactivity and stability of selected flavor compounds. J Food Drug Anal 23:176-190

Worfel RC, Schneider KS, Yang TCS (1997) Suppressive effect of allyl isothiocyanate on populations of stored grain insect pests. J Food Process 21:9-19 
Wu X, Zhou QH, Xu K (2009) Are isothiocyanates potential anti-cancer drugs? Acta Pharmacol Sin 30:501-512

Wylie FR, Janssen-May S (2016) Red imported fire ant in Australia: what if we lose the war? Ecol Manag Restor 18:32-44

Wylie FR, Yang C-CS, Tsuji K (2020) Invader at the gate: the status of red imported fire ant in Australia and Asia. Ecol Res 35:6-16
Zettler JL, Arthur FH (2000) Chemical control of stored product insects with fumigants and residual treatments. Crop Prot 19:577-582

Publisher's Note Springer Nature remains neutral with regard to jurisdictional claims in published maps and institutional affiliations. 